\title{
Editorial
}

\section{Early adversity, stress, and neurobehavioral development}

\author{
Megan R. Gunnar \\ Institute of Child Development, University of Minnesota, Minneapolis, MN, USA
}

\section{There is a Future in the Past}

In 1956, Seymour Levine and colleagues (Levine, Chevalier, \& Korchin, 1956) took a group of infant rats and subjected them to mild shocks every day. They then compared the pups' ability to learn in adulthood to two groups: one group that was handled but not shocked, and one group that was not handled nor were their nests disturbed. This was one of the first experimental studies of early adversity and the results were shocking (no pun intended). The group that was impaired in learning was the group who had not been handled or shocked. The handled-shocked and the handled-only group did equally well on every measure. Thus was born the research area known as infantile stimulation. Infant stimulation was shown to alter behavior into adulthood, to be most effective during a sensitive period in the first two weeks of life, to reduce fearfulness, increase stress resistance, and delay immune senescence. When President Johnson declared a war on poverty and had Sargent Shriver convene the task force that created Head Start, the work on infantile stimulation, among others, supported the idea that infancy and early childhood was a critical period for providing stimulation.

The fact that electric shocks produced positive effects on development seems to fly in the face of our current attempts to explain why early adversity has long-term negative impacts on human development. Perhaps the problem is that rats and mice are not good models of humans. In this case, that is unlikely. Recall the rodent infantile stimulation work was paralleled by the cultural anthropology of Landauer and Whiting (1964) who showed more robust physical growth in cultures that exposed their infants to various forms of stress from circumcision to cold baths. There is a great deal of excellent food for thought in that early literature as we attempt to understand how early adversity "gets under the skin" to influence long-term outcomes in human development.

Some of the themes and findings from that early work reappear in the pages of this special issue, although frequently not attributed to these early origins. For example, the early literature identified stimulation as having a U-shape function in relation to adult outcomes: too little and too much was associated with poorer outcomes (Denenberg, 1964). We have rediscovered this in the theorizing of Boyce and Ellis (2005) who argue that too

Author for Correspondence: Megan R. Gunnar, Institute of Child Development, University of Minnesota 51 East River Road, Minneapolis, MN 55455, USA; E-mail: gunnar@umn.edu.

Cite this article: Gunnar MR (2020). Early adversity, stress, and neurobehavioral development. Development and Psychopathology 32, 1555-1562. https://doi.org/ $10.1017 /$ S0954579420001649 little and too much adversity are both associated with higher stress reactivity, while a moderate amount of adversity within the child's ability to cope is associated with reduced, but not hypoactive reactivity. An example of issues that the early rodent work highlighted that we should revisit but have not is the possibility that noxious stimulation has opposing effects at different points in development. Thus, the same levels of shock that in preweaning rat pups results in less fearful behavior, when delivered to postweaning animals impairs functioning (Denenberg, 1964). That early work is reminiscent of current work by Regina Sullivan showing that in infant rat pups pairing a novel odor with shock results in a preference for and approach to the odor if the dam is present and an aversion when she is absent, while in older animals only an aversion is observed (see review Hostinar, Sullivan, \& Gunnar, 2014). While this might seem unlikely in humans, Nim Tottenham and colleagues (Tottenham, Shapiro, Flannery, Caldera, \& Sullivan, 2019) have recently shown something quite similar in preschool-age children. After being conditioned to identify one stimulus with a noxious event, preschool-aged children actually showed a preference for that stimulus when the parent was present, but avoided it when the parent was not present. These data indicate that the attachment figure moderates how aversive stimuli impact the young child, but perhaps not the older child, as will be discussed.

Another theme in the early work was that the effects of infantile stimulation were likely mediated by what it did to parental care. Specifically, when the pup was either handled or shocked it was returned to the dam in an agitated state. That elicited heightened nurturing stimulation (licking and grooming) by the dam which in reality was the critical feature of the infant's experience (Smotherman \& Bell, 1980). While sometimes controversial (Denenberg, 1999), it is largely accepted that stimuli as disparate as handling and shock produce similar outcomes because they both result in heightened maternal care. Similarly, the early adversity literature in human development converges on parental care as the central protective factor and the process that, when it fails the child, becomes the main conduit for adversity to impact the child's development. These themes from the early animal literature have strong resonance in our current work on early adversity, stress, neurobehavioral development, and resilience. In what follows I will outline many of the current issues with which the field is grappling and point to papers in this special issue that reflect these issues.

\section{The Nature of the Stimulus}

Early-life stress, adverse childhood experiences, early adversity: these largely interchangeable constructs refer to a wide range of 
experiences and contexts that are non-optimal for human development. Physical, sexual, and psychological abuse, neglect, poverty, institutional (orphanage) care, exposure to violence in the home and community, and bullying have all been studied (Rutter, 2016). All are associated with poor physical and psychological health with frequent or chronic activation of stress biology presumed to play a mediating role. Since the advent of developmental studies of adversity, psychologists have grappled with how to understand the relations between what children experience and how those experiences affect them. As a field we learned that there is not a one-to-one relationship between a given type of adversity and a given outcome, which borrowing from general systems theory we termed multifinality (von Bertalanffy, 1969). We also learned that different adverse conditions can be associated with the same outcome, equifinality (von Bertalanffy, 1969). We learned that the accumulation of adversities better predicts the probability of poor functioning in adulthood than the presence of any given adversity.

We have long defined early-life stress and adversity as the presence of stimuli that impair development and/or the absence of stimuli that are needed to support typical development. Recently, McLaughlin and Sheridan (2016) have formalized this distinction, arguing that there are two dimensions of risk or adversity underlying these early experience effects: threat and deprivation. Both can be thought of as existing along continua from low to high. Several tests of this model have recently provided supportive evidence. More importantly, McLaughlin and Sheridan (2016) argue that learning, in addition to stress pathways, is critical for the transduction of early adversity into developmental outcomes. Milojevich, Machlin, and Sheridan (this issue) pit early adversity, assessing threat and deprivation as separate dimensions, against parental socialization in predicting emotion regulation among diverse 7 -year-olds. They find that parental socialization in the form of the parents' reports about their own emotion regulation is a more powerful predictor and absorbs the variance obtained from threat and deprivation as predictors. Because socialization was defined in this paper as parental emotion regulation, it is of course possible that genetic contributions to parent and child emotion regulatory abilities and not learning, per se, were the operative factors.

One wonders, however, whether threat and deprivation are the only dimensions that we need to examine. In studies of what activated the hypothalamic-pituitary-adrenocortical (HPA) axis, for example, it is the combination of threats to the social self and unpredictability/uncontrollability that are critical. Predictability/ controllability are key facets of coping, in that it is tremendously difficult to cope without being able to predict and control potentially threatening events. Tarullo, Tuladhar, Kao, Drury, and Meyer (this issue) provide a lovely example of this by decomposing socioeconomic status into parental education, income-to-needs, food insecurity, family chaos, and neighborhood risk. They also measure both hair cortisol and diurnal salivary rhythm in infants and 3.5-year-olds. The results suggest that both lower parent education and measures reflecting greater unpredictability were associated with higher cortisol levels and altered daily rhythms. These data suggest that in addition to deprivation and threat, our models of early-life adversity need a third dimension of unpredictability/uncontrollability. Adding this dimension would not only be consistent with the work of Dickerson and Kemeny (2004), but also of the work of Baram et al. (2012) who have shown that fragmented and unpredictable stimulation during early development impairs cognitive development and increases the risk of mental disorders in rodent models and in humans. Recently this group has also shown that fragmented/unpredictable maternal behavior in infancy predicts a blunted cortisol response to pain stressors in the baby (Norona-Zhou et al., 2020).

\section{Systems that are Involved}

If adversity early in life influences physical and mental health in adulthood, what are the mechanisms and processes linking these early experiences to later outcomes? This is, of course, one of the primary questions in this field of study. The truth of the matter is that, in addition to psychosocial processes, stress activates many cascading physiological and molecular systems, all of which probably play some role in transducing stressful experiences into long-term outcomes (Dhama et al., 2019; Joëls \& Baram, 2009). The HPA system has been the most studied as a system shaped by stress and one that mediates stressor impacts on development. A number of the papers in this issue measure activity of this neuroendocrine system. This includes the paper by $\mathrm{R}$. Perry and colleagues which shows that activity of the HPA axis is a necessary but not sufficient mechanism in translating adverse experiences to infant outcomes. It also includes the paper by VanZomeren and colleagues (this issue), which found that maltreatment after age 5 but not before age 5 was associated with a reduced diurnal cortisol rhythm in school-aged children.

The immune system, most notably inflammatory activity of the immune system, has long been studied in the animal literature in relation to early-life stress (Coe, Rosenberg, \& Levine, 1988); however, it has only more recently become a focus of human developmental research on adversity and development (Miller, Chen, \& Parker, 2011). The immune, HPA, and sympathetic systems all respond to acute stressors and alter their activity in the context of chronic stress; furthermore, they all influence one another (Engel \& Gunnar, 2020). Several papers in the present issue include measures of inflammatory cytokines, but it is the paper by Reid and Danese (this issue) that provides a very useful review of research on early-life adversity and inflammation that challenges some of our assumptions and may lead us to refine our hypotheses.

Of course, as the late, great Bruce McEwen would want to remind us, the brain is the central organ of stress, health, and disease (McEwen, 2013). Certainly, there is increased interest in understanding how stress impacts brain development, and thus how alterations in neural systems may mediate the long-term impact of early-life adversity (Morin et al., this issue; Tottenham, 2020). Tottenham (this issue) provides a fascinating and generative argument linking early caregiving adversity to later outcomes as mediated by medial prefrontal cortex (mPFC) to amygdala pathways. Blaisdell, Barker, Guiliano, and Fisher (this issue) examine frontal and parietal electroencephalography (EEG) asymmetry in foster care versus community 3 -year-olds and find, perhaps unexpectedly, that the community children living in low-income neighborhoods exhibit significant parietal asymmetry with evidence of greater activity over the right than left parietal regions. This is a pattern which in adults is associated with both reduced arousal (e.g., less caffeinated) and an increased history of major depression (Steward, Towers, Coan, \& Allen, 2011). While there may well be developmental changes in the associations between EEG asymmetry and psychological functioning, it is interesting that in the Blaisdell article (this issue) overall power over both left and right parietal regions was lower (i.e., activation high) in the foster care 
versus the community sample. This was also a pattern noted in a study my group did a number of years ago examining parietal functioning and memory in youth adopted from orphanage/ institutional settings (Guler et al., 2012).

\section{Sensitive Periods}

When we study children it is often difficult to isolate the developmental period when adversity started and ended. This makes it very difficult to determine if there are sensitive periods, always recognizing that there may be different sensitive periods for different systems. If we focus, though, on stress-reactive systems, we can perhaps use the animal literature and several natural experiments to ask whether there are sensitive periods for these systems. In this issue there are papers dealing with sensitive periods shaping the HPA systems and the immune system.

In the rodent literature there is clear evidence of a sensitive period for shaping fearfulness and the HPA axis in the first 8to 10-days of life (Gunnar \& Vazquez, 2006). The challenge in translating this to humans is that this roughly corresponds to the last trimester of pregnancy and perhaps the first few months postnatal. Thus, the parenting adversity literature in rodent pups might actually translate better to prenatal than postnatal stress in humans. Indeed, there is growing evidence of hyper-reactivity of the HPA axis following prenatal stress in human development (see review, Zijlmans, Riksen-Walraven, \& de Weerth, 2015). The review paper by Davis and Narayan (this issue) provides a broader review of the pregnancy period as a unique window of opportunity for understanding and hopefully deterring the intergenerational transmission of adversity and mental health problems. The paper by Howland, Sandman, Poggi Davis, and Glynn (this issue) focuses on maternal psychological distress, one form of prenatal stress, and its impacts on fetal trajectories. If trajectories of fetal development are altered by the mother's psychological distress, this certainly could be one factor linking prenatal stress to later psychosocial and physical health.

While the Davis and Narayan and the Howland and colleagues' papers deal with the prenatal period, several papers focus on other potential periods when life stress may have especially powerful impacts on development. Doom et al. (this issue) examined cardiovascular health as it is impacted by stresssensitivity systems activated and shaped in response to adversity at different points in development. Using a large cohort of children from Chile, they examined young adult cardiometabolic health as a function of stressful life circumstances in infancy, and at age 5, 10, and adolescence. Supporting an early sensitive period, infancy was associated with cardiovascular risk measures over and above stressors assessed at any of the other later periods.

In my own work we have examined the impact of early institutional care on children's HPA axis reactivity and found that even when adopted as toddlers, the axis exhibits blunted levels of activity and reactivity for years postadoption (Koss, Hostinar, Donzella, \& Gunnar, 2014). This is consistent with evidence from the Bucharest Early Intervention Project (BEIP), which suggests that removal from institutional care after, but not before, age 2 is associated with a blunting of the axis's response to stressors for years post removal (McLaughlin et al., 2015).

In addition, the current author's lab examined whether the HPA axis during the peripubertal period is capable of recalibrating to current life conditions. It was found that youth adopted internationally from institutions had blunted cortisol responding early in puberty; however, with time over the pubertal development period, cortisol reactivity became comparable to that in youth who did not have early adverse histories (Gunnar, DePasquale, Reid, Donzella, \& Miller, 2019). Importantly, in the paper by Wade et al. (this issue) using the BEIP population, concurrent care quality did not override the impact of early institutional care in predicting either cortisol or pre-ejection period (PEP) responses to the same stressor we used (i.e., the Trier Social Stress Test). Notably, however, it seems likely that most of the children were experiencing a poorer quality of parental care than were the youth in our sample, as in the BEIP study families received an average rating of Mixed (adequate but with some evidence of harshness), while our analyses of families who adopt internationally tends to find that nearly all tend to be adequately to highly sensitive and responsive. This raises the question of how good it needs to get before stress systems recalibrate to less blunted, more reactive activity at puberty.

In the paper by Perry, DePasquale, Donzella, and Gunnar (this issue) we explored whether the increase (normalization) of cortisol reactivity among the previously institutionalized youth was associated with an increase or decrease in internalizing and/or externalizing symptoms. Our initial expectation was that if the HPA system was responding more normally this might be associated with a reduction in externalizing symptoms, which we had previously found to be related to blunted cortisol reactivity in children with similar histories (Gunnar et al., 2019). However, this is not what N. Perry and colleagues found. She found that recalibration of stress systems from blunted to responsive with increasing pubertal stage may create emotion regulatory challenges for the adolescent that increase anxiety and depressive symptoms and further heightened cortisol reactivity.

\section{Individual Differences in Vulnerability and Resilience}

No matter what the type or degree of adversity, children differ in their response. When adversity is severe, few escape some impact, but still many manage to survive and have lives of accomplishment (see Russotti, Warmingham, Handley, Rogosch, \& Cicchetti, this issue). We have known this going back as early as the Kauai longitudinal study (Werner, 1996; Werner, Bierman, \& French, 1971). Yet even when individuals appear psychologically and academically resilient, that resilience may only be skin deep. Measures of physical health and allostatic load sometimes reveal marked effects, perhaps not only of the adversity, but of the costs of overcoming it (Brody et al., 2013). Russotti et al. (this issue) examined competence and maladaptation across multiple domains for young adults from lowincome backgrounds who had or had not been maltreated in childhood. While maltreatment decreased the likelihood of multidomain competence, those black men who achieved across-the-board competence by late adolescence also carried a higher inflammatory burden as indexed by levels of C-reactive protein. For some, clearly, there is a cost of achieving despite the odds. These findings on risk and resilience reveal two aspects of individual differences that we need to understand. Why are some individuals more sensitive to adversity than others, and for any given individual, which systems will be the most impacted?

Genetics doubtlessly underlie some of the individual differences in the effects of early adversity. In this issue, Chen and colleagues operating from a classic diathesis-stress model, describe novel ways, using big data approaches, to identify the genotypes that increase vulnerability of the fetus to maternal depression during pregnancy. Recently, in developmental science, diathesisstress models of individual differences have given way to the 
differential susceptibility hypothesis (Belsky \& Pluess, 2009), which argues that genetic alleles that increase vulnerability in some contexts actually increase positive outcomes in others. The other related argument is termed Biological Sensitivity to Context (Boyce \& Ellis, 2005), which argues that individuals who are more physiologically reactive thrive in supportive environments but are at risk for poor outcomes in adverse ones. None of the articles in the present issue explicitly addresses either of these individual difference hypotheses. However, the article by Herzberg, Hunt, Thomas, and Gunnar (this issue) comes close. This paper describes a new version of the Trier Social Stress Test that is appropriate for children and adolescents and is designed for the magnetic resonance imaging (MRI). The goal of the Minnesota Imaging Stress Test in Children (MISTiC) is to allow an examination of brain activation in association with HPA axis reactivity in children and adolescence. In the paper in this issue, the authors also explore differences in activation patterns for individuals who do and do not respond to the MISTiC. This should give us a method to understand the neural response differences in individuals who are more or less impacted by acute stress conditions, at least those that threaten the social self.

\section{Sex/Gender Differences}

From the early rodent studies it was apparent that effects on males and females differed. This is an area of individual differences in human development that we are still struggling to both describe and understand. Males seem to be more vulnerable to prenatal and early postnatal stress (e.g., Sandman, Glynn, \& Davis, 2013). Whether preadolescent children show sex differences in the reactivity of stress-responsive systems is not clear, with many studies yielding similar responses for boys and girls (Gunnar \& Vazquez, 2006). Multisystem studies of the relation between HPA and sympathetic nervous system (SNS) reactivity, such as the study by Hagan, Roubinov, Boyce, and Bush (this issue) also point to the fact that physiological reactivity may bear different associations to behavior (e.g., aggression) for boys and girls. It also appears to be the case that by adolescence stress reactivity and its patterning across systems has clearly diverged, as noted by Ho, Pham, Miller, Kircanski, and Gotlib (this issue). It is also the case that what creates stress for males and females begins to diverge at least as early as adolescence, with males being more responsive to performance stressors and females to social rejection stressors (e.g., Stroud et al., 2009; Stroud, Salovey, \& Epel, 2002). While some of this may well be due to gender socialization (Myrsten et al., 1984), we also need to better understand the interaction between gonadal and adrenal steroids as Shirtcliff and colleagues have argued (Marceau et al., 2014). In addition, we need to understand whether the interaction of stress and sex steroid regulatory systems is involved in pathways from adversity to depression (female prevalent outcome) versus conduct problems (male prevalent outcome).

\section{Parent as Buffer and Conduit}

Humans are social animals who rely heavily on relationships with others to manage stress (Beckes \& Coan, 2011). This is especially true for young children who cannot cope with adversity on their own, a fact that is reflected in attachment as a species typical behavior (Bowlby, 1969). The most devastating form of early-life stress, then, is the failure of adult caregivers to protect, nurture and provide for infants and young children. In her theoretical paper (this issue) Nim Tottenham provides an account that relates caregiving support to the healthy development of emotion and stress regulation via mPFC-amygdala circuitry. This account also, of course, describes how caregiver adversity underlies dysregulated development of mPFC-amygdala circuity as the developing child attempts to manage threats and challenges without sufficient support and/or where the support figure is the source of the threat.

When the attachment relationship is secure, the parent provides a secure base for exploration and regulation of psychological and somatic stress. The parent acts as a safety signal that can allay fear, but even when the child remains frightened of some offending event, the parent's presence reduces or prevents activation of the HPA axis (Nachmias, Gunnar, Mangelsdorf, Parritz, \& Buss, 1996). Parenting causally impacts the HPA axis, as shown in the Raby, Bernard, Gordon, and Dozier (this issue) paper demonstrating that a parenting intervention known to improve atttachment security also improves the diurnal cortisol rhythm in internationally-adopted children. The child's physiology may also mirror (be attuned) with the parent's physiological responses to stimulation, for better or worse (see DePasquale, this issue). The attachment figure remains a potent safety signal at least until midway in pubertal development (Doom, Hostinar, VanZomeren-Dohn, \& Gunnar, 2015). When the parent's presence and availability buffers activation of stress-responsive systems, we term this parental social buffering. Parents may also protect children from stress by serving as an instrumental buffer, taking less for themselves so that their children may have more in cases of poverty, for example. Or, shielding their children from frightening local, national, or international events by staying calm and not catastrophizing in front of the children.

Because the parent is a conduit for stress to the child, how the parent reacts to external stress may be a critical factor in how adverse conditions impact the child. For example, stress on the parent impairs the parent's ability to provide sensitive, responsive, supportive care, and guidance (Deater-Deckard, 2004). However, when parents can manage to provide positive parenting in the face of chronic stressors such as poverty, children can thrive. We have shown this in a study of children undergoing well-child exams with inoculations. Parents who managed to create secure attachment relationships with their toddlers despite living near or below the federal poverty limit, had children whose cortisol levels during the doctor's visit were low, while insecurely attached children showed higher cortisol levels than those shown by children who were not living in poverty (Johnson, Mliner, Depasquale, Troy, \& Gunnar, 2018). Brown et al. (this issue) have provided an even more satisfying example because they directly examined parenting in a Head Start sample. They showed that positive parenting was associated with less of a cortisol increase to a laboratory stressor task and that the effect was more marked among families experiencing greater poverty. Both our earlier study and that of Brown and colleagues raise the possibility that positive parenting in the context of high adversity creates an even greater capacity of the parent to buffer activity of the child's stress-responsive physiological systems than positive parenting in less adverse contexts.

Supportive parenting early in life may also provide a shield against the impacts of stressors experienced later in development. This was demonstrated in the paper by Tang et al. (this issue). Studying the adolescents from the BEIP they examined stress at several points in adolescence in relation to measures of inflammation. The youth who were in their care-as-usual group and had thus spent their infancy and early childhood in institutional care were more inflamed if they experienced more stress in 
adolescence. This was not the case for the youth who had been removed from institutional care and placed in supportive foster homes. Although not examined in this paper, it seems likely that children placed in foster homes relative to those left in institutional care developed better coping and emotion regulatory strategies that allowed them to weather later stressors with less adverse biological impact.

If the parent is a conduit as well as a buffer, then the parent can amplify the stress the child experiences as well as reduce it. The paper by Hostinar's group (Parenteau et al., this issue) provides an example. Previously, when Professor Hostinar was a student in my group she showed that the presence of the parent prevented elevations in cortisol among 9- and 10-year old children (Hostinar, Johnson, \& Gunnar, 2015). In the Parenteau et al. paper (this issue) they found that this was the case when parents were less well educated, but not when they were highly educated. The stressor was the Trier Social Stress Test for Children (TSST-C), a social-evaluative performance stressor. The more highly educated parents were also higher in state anxiety, perhaps worried that their children would not perform well. The parent's anxiety about the child's performance may have been conveyed to the child. This finding is comparable to evidence in the study by Nachmias et al. (1996), which showed that when the mothers of 18-month-olds pushed them to approach things they found scary, this was associated with insecure attachment and a failure to buffer the HPA axis. Thus, the parent is both a buffer and a conduit.

\section{Discrimination and Stigma}

Parents, teachers, and the other adults in a child's life may have additional learning objectives when raising children from groups who are discriminated against in society. Lupien et al. (this issue) examine whether stigma linked with having a family member with mental illness versus cancer is associated with depressed symptoms and altered cortisol activity in family members. They found that even though family members experienced greater negative evaluation by others, depressive symptoms and HPA axis alterations were not noted. This is unlike the case for racial discrimination.

There is good evidence that racial discrimination is a source of stress that can impair health and affect stress-responsive physiological systems as discussed by Adam, Hittner, Thomas, Villaume, and Nwafor (this issue). Positive ethnic and racial identification can protect against perceived racial discrimination and regulate the HPA axis. This may be especially important during adolescence when the child moves away from the family's protection into the broader social arena. While parents are essential in helping children develop a positive sense of their own racial and ethnic identity, among adolescents and emerging adults, peers also begin to play a critical role (Nelson, Syed, Tran, Hu, \& Lee, 2018). Given the damaging effect of discrimination, understanding the mechanisms that transduce the experience and perception of discrimination into physical and mental health problems is critical. As indicated in Adam et al. (this issue), these insights can also point to protective processes that are malleable and thus to interventions that will improve developmental health.

\section{Beyond Admiring the Problem: What Can a Neurobiological Perspective Tell Us?}

As this last point indicates, understanding how adversity "gets under the skin" and shapes mental and physical health during development can point to protective processes that are malleable and thus can be targets of intervention. Parenting is one we have already discussed and is perhaps the most critical for young children. A positive racial-ethnic identity is a malleable target. In this issue, however, many of the papers have brought a multilevel, neuroscience, and stress physiology perspective to the problem of early-life adversity. We have asked what changes "under the skin" when children are reared under conditions of adversity. While this work has helped us understand mechanisms, how important is it to informing how we can address and mitigate the impact of childhood adversity? Two of the papers in this issue tackle this question. Pollak and Wolfe (this issue) argue that neuroscience measures can be harnessed to not just examine but to address the problem of childhood poverty. They make a number of arguments, but two seem especially pertinent. In one section they argue that brain physiology at times can predict behavior better than available behavioral measures. It seems unlikely in the near future that we will use imaging on a population basis to identify children who need intervention. However, it does seem possible that imaging may help us identify neural responses that are predictive of who needs intervention. Once identified then we may be able to develop or expand on the tasks that elicited the predictive neural responses and deploy those as more refined and subtle behavioral measures that capture emerging deficits in key capacities. In another section, Pollak and Wolfe (this issue) argue that neural measures might one day allow us to evaluate interventions and policies earlier. This point is related to the first. To the extent that brain measures can detect the processes that will lead to the behavioral targets of the intervention or policy before the behavior emerges, then using brain measures might allow for more rapid analysis of whether what we are doing is working. In adaptive interventions, such measures might allow for a more rapid adjustment and personalizing of the intervention.

Obradović and Armstrong-Carter (this issue) take a similar approach to asking whether and how the assessment of stress physiology in educational settings might be leveraged to aid in evaluating educational interventions to enhance the school success of every child. They note that stress physiology has not been measured in educational settings that often, making the case that this is a missed opportunity. Their goal is to stimulate a new wave of research that will advance the science of developmental stress research and, ultimately improve educational policy and practice.

\section{Conclusions}

As the papers in this issue attest, what started with some smallscale studies of rat pups has burgeoned into a major topic of work in our field. Demonstrating that early-life stress has significant impacts on neurobiology and physical, as well as mental, health has brought widespread attention to the problem of childhood adversity by policy makers around the world. We are still a long way from understanding the various ways that nature transduces adversity into developmental outcomes. Everyone who has sought to understand adversities' impacts by studying stresssensitive physiological and neurobiological systems knows, or will learn, that the systems they are measuring are dynamic and do not give up their secrets easily. There are some groups, including one I am a part of, that are trying to develop batteries of hormonal and molecular measures that can be easily deployed in settings like pediatric offices to reliably identify infants and young children who are being impacted by excessive stress. It is too early to determine whether we will be successful; however, it is clear that the more we as a field show that stress and adversity 
"gets under the skin" and alters development, the more there will be an appetite for assessing systems that transduce adversity to under the skin effects before they can result in poor physical and mental health. Many, if not most, of the adversity that impacts children can be traced to structural issues, such as poverty, inequality, and discrimination. While fixing the root of the problem of earlylife stress would seem to be the most logical way to improve outcomes for children and society, it is not likely to be achievable in the near or even perhaps the long term. This leaves it to us to understand and intervene to support the people and processes that can protect children from adversity and effectively treat children whose stress-buffers have failed. There is still much work to do.

\section{Final Thoughts and Acknowledgements}

The special section in this issue are the papers from the 2019 Minnesota Symposium on Child Psychology which my colleagues elected to be on early-life adversity, stress, and neurobehavioral development to honor my 9 years as the Director of the Institute of Child Development. I am very grateful to them for this honor and for the two days of great intellectual stimulation the symposium afforded. I am especially grateful to my former student, Nim Tottenham, who co-led the symposium with me and was my co-editor on the symposium section of this issue. I am also very appreciative that my colleague, Dante Cicchetti, was able to arrange this special issue of Development and Psychopathology to augment the symposium so that more voices and perspectives could be represented. I am also very grateful to the many authors who produced such wonderful papers.

As this editorial was written during spring and summer of 2020 while we sheltered-in-place during the COVID-19 pandemic, I am overwhelmingly aware of how the inequalities that produce the early adversities we study are being amplified by the pandemic we are all living through. Sitting in Minnesota, I cannot forget that the murder of George Floyd ripped the scales from the eyes of many of us who experience white privilege so that we finally see the structural racism that feeds the inequalities the virus has exploited. It seems very timely but also somehow inadequate to be putting out a special issue devoted to this topic. While we arm ourselves with knowledge as scientists and try to use that science to help children and families, this may be a time to also do more. While as noted, changing the structural factors that create adverse conditions for so many of the world's children is likely to take more than our own lifetimes, it is time to commit ourselves to more directly translate our work to policies that confront these structural problems of race and class.

Acknowledgment. I would like to thank Carrie DePasquale, who departed this world on September 12, 2020, for her careful editing of the draft of this editorial.

Funding Statement. This editorial to the special issue was supported by grant number HD 095904 and The JPB Foundation through a grant to The JPB Research Network on Toxic Stress: A Project of the Center on the Developing Child at Harvard University as well as a grant from the Bezos Family Foundation.

\section{Conflicts of Interest. None}

\section{References}

Adam, E. K., Hittner, E., Thomas, S., Villaume, S. C., \& Nwafor, E. (this issue). Racial discrimination and ethnic racial identity in adolescence as modulators of HPA-axis activity. Development and Psychopathology, 32, 1669-1684.
Baram, T. Z., Davis, E. P., Obenaus, A., Sandman, C. A., Small, S., Solodkin, A. \& Stern, H. (2012). Fragmentation and unpredictability of early life experience in mental disorders. American Journal of Psychiatry, 169, 907-915.

Beckes, L., \& Coan, J. A. (2011). Social baseline theory: The role of social proximity in emotion and economy of action. Social and Personality Psychology Compass, 5, 976-988.

Belsky, J., \& Pluess, M. (2009). Beyond diathesis stress: differential susceptibility to environmental influences. Psychological Bulletin, 135, 885-908.

Blaisdell, K. N., Barker, T. V., Guiliano, R. J., \& Fisher, P. A. (this issue). Alpha electroencephalogram (EEG) asymmetry among toddlers in foster care. Development and Psychopathology, 32, 1743-1753.

Bowlby, J. (1969). Attachment and loss: Attachment (Vol. 1). New York: Basic Books.

Boyce, W. T., \& Ellis, B. J. (2005). Biological sensitivity to context: I. An evolutionary-developmental theory of the origins and functions of stress reactivity. Development \& Psychopathology, 17, 271-301.

Brody, G. H., Yu, T., Chen, E., Miller, G. E., Kogan, S. M., \& Beach, S. R. (2013). Is resilience only skin deep? Rural African Americans' preadolescent socioeconomic status-related risk and competence and age 19 psychological adjustment and allostatic load. Psychological Science. e-pub ahead of print.

Brown, S. M., Schlueter, L. J., Hurwich-Reiss, E., Dmitrieva, J., Miles, E., \& Watamura, S. E. (this issue). Parental buffering in the context of poverty: Positive parenting behaviors differentiates young children's stress reactivity profiles. Development and Psychopathology, 32, 1778-1787.

Chen, L. M., Tollenaar, M. S., Hari Dass, S. A., Bouvette-Turcot, A. A., Pokhvisneva, I., Gaudreau, H., ... members of the MAVAN Study Team. (this issue). Maternal antenatal depression and child mental health: Moderation by genomic risk for attention-deficit/hyperactivity disorder. Development and Psychopathology, 32, 1810-1821.

Coe, C. L., Rosenberg, L. T., \& Levine, S. (1988). Immunological consequences of psychological disturbance and maternal loss in infancy. In C. Rovee-Collier \& L. P. Lipsitt (Eds.), Advances in infancy research (Vol. 5, pp. 97-134). Norwood, N.J.: Ablex.

Davis, E. P., \& Narayan, A. J. (this issue). Pregnancy as a period of risk, adaptation, and resilience for mothers and infants. Development and Psychopathology, 32, 1625-1639.

Deater-Deckard, K. D. (2004). Parenting stress. New Haven, Connecticut: Yale University Press.

Denenberg, V. H. (1999). Commentary: Is maternal stimulation the mediator of the handling effects in infancy? Developmental Psychobiology, 34, 1-3.

Denenberg, V. R. (1964). Critical periods, stimulus input, and emotional reactivity: A theory of infantile stimulation. Psychological Review, 71, 335-351.

DePasquale, C. E. (this issue). A systematic review of caregiver-child physiological synchrony across systems: Associations with behavior and child functioning. Development and Psychopathology, 32, 1754-1777.

Dhama, K., Latheef, S. K., Dadar, M., Samad, H. A., Munjal, A., Khandia, R., ... Joshi, S. K. (2019). Biomarkers in stress related diseases/disorders: Diagnostic, prognostic, and therapeutic values. Frontiers in Molecular Bioscience, 18, 1-47.

Dickerson, S. S., \& Kemeny, M. E. (2004). Acute stressors and cortisol responses: A theoretical integration and synthesis of laboratory research. Psychological Bulletin, 103, 355-391.

Doom, J. R., Hostinar, C. E., VanZomeren-Dohn, \& Gunnar, M. R. (2015). The roles of puberty and age in explaining the diminished effectiveness of parental buffering of HPA reactivity and recovery in adolescence. Psychoneuroendocrinology, 59, 102-111.

Doom, J. R., Rivera, K. M., Blanco, E., Burrows, R., Correa-Burrows, P., East, P. L., ... Gahagan, S. (this issue). Sensitive periods for psychosocial risk in childhood and adolescence and cardiometabolic outcomes in young children. Development and Psychopathology, 32, 1864-1875.

Engel, M. L., \& Gunnar, M. R. (2020). The development of stress reactivity and regulation during human development. International Review of Neurobiology, 150, 41-76.

Guler, O. E., Hostinar, C. E., Frenn, K. A., Nelson, C. A., Gunnar, M. R., \& Thomas, K. M. (2012). Electrophysiological evidence of altered memory processing in children experiencing early deprivation. Developmental Science, 15, 345-358.

Gunnar, M. R., DePasquale, C. E., Reid, B. M., Donzella, B., \& Miller, B. S. (2019). Pubertal stress recalibration reverses the effects of early life stress 
in postinstitutionalized children. Proceedings of the National Academy of Sciences, 116, 23984-23988.

Gunnar, M. R., \& Vazquez, D. (2006). Stress neurobiology and developmental psychopathology. In D. Cicchetti \& D. Cohen (Eds.), Developmental psychopathology: Developmental neuroscience (2nd ed., Vol. 2, pp. 533-577). New York: Wiley.

Hagan, M. J., Roubinov, D. S., Boyce, W. T., \& Bush, N. R. (this issue). Associations between multisystem stress reactivity and peer nominated aggression in early childhood vary by sex. Development and Psychopathology, 32, 1888-1898.

Herzberg, M. P., Hunt, R. H., Thomas, K. M., \& Gunnar, M. R. (this issue). Differential brain activity as a function of social evaluative stress in early adolescence: Brain function and salivary cortisol. Development and Psychopathology, 32, 1926-1936.

Ho, T. C., Pham, H. T., Miller, J. G., Kircanski, K., \& Gotlib, I. H. (this issue). Sympathetic nervous system dominance during stress recovery mediates associations between stress sensitivity and social anxiety symptoms in female adolescents. Development and Psychopathology, 32, 1914-1925.

Hostinar, C. E., Johnson, A. E., \& Gunnar, M. R. (2015). Parent support is less effective in buffering cortisol stress reactivity for adolescents compared to children. Developmental Science, 18, 281-297.

Hostinar, C. E., Sullivan, R. M., \& Gunnar, M.R.. (2014). The psychobiological mechanisms underlying social buffering of the hypothalamic-pituitaryadrenocortical axis: A review of animal models and human studies across development. Psychological Bulletin, 140(1), 256-292.

Howland, M. A., Sandman, C. A., Poggi Davis, E., \& Glynn, L. M. (this issue). Prenatal maternal psychological distress and fetal developmental trajectories: Associations with infant temperament. Development and Psychopathology, 32, 1685-1695.

Joëls, M., \& Baram, T. Z. (2009). The neuro-symphony of stress. Nature Reviews Neuroscience, 10, 459-466.

Johnson, A. B., Mliner, S. B., Depasquale, C. E., Troy, M., \& Gunnar, M. R. (2018). Attachment security buffers the HPA axis of toddlers growing up in poverty or near poverty: Assessment during pediatric well-child exams with inoculations. Psychoneuroendocrinology, 95, 120-127.

Koss, K. J., Hostinar, C. E., Donzella, B., \& Gunnar, M. R. (2014). Social deprivation and the HPA axis in early development. Psychoneuroendocrinology, $50,1-13$.

Landauer, T. K., \& Whiting, J. W. M. (1964). Infantile stimulation and adult stature of human males. American Antropologist, 66, 1007-1028.

Levine, S., Chevalier, J. A., \& Korchin, S. J. (1956). The effects of early shock and handling on later avoidance learning. Journal of Personality, 24, 475-493.

Lupien, S. J., Roy, D. C., Raymond, C., Leclaire, S., Wan, N., Labelle, R., ... Ouellet-Morin, I. (this issue). Stigma associated with parental depression or cancer: Impact on spouse and offspring's cortisol levels and socioemotional functioning. Development and Psychopathology, 32, 1822-1837.

Marceau, K., Shirtcliff, E. A., Hastings, P. D., Klimes-Dougan, B., Zahn-Waxler, C., Dorn, L. D., \& Susman, E. J. (2014). Within-adolescent coupled changes in cortisol with DHEA and testosterone in response to three stressors during adolescence. Psychoneuroendocrinology, 41, 33-45.

McEwen, B. S. (2013). The brain on stress: Toward an integrative approach to brain, body, and behavior. Perspectives in Psychological Science, 8, 673-675.

McLaughlin, K. A., \& Sheridan, M. A. (2016). Beyond cumulative risk: A dimensional approach to childhood adversity. Current Directions in Psychological Science, 25, 239-245.

McLaughlin, K. A., Sheridan, M. A., Tibu, F., Fox, N. A., Zeanah, C. H., \& Nelson, C. A. (2015). Causal effects of the early caregiving environment on development of stress response systems in children. Proceedings of the National Academy of Sciences, 112, 5637-5642.

Miller, G. E., Chen, E., \& Parker, K. J. (2011). Psychological stress in childhood and susceptibility to the chronic diseases of aging: Moving toward a model of behavioral and biological mechanisms. Psychological Bulletin, 137, 959-997.

Milojevich, H. M., Machlin, L., \& Sheridan, M. A. (this issue). Early adversity and children's emotion regulation: Differential roles of parent emotion regulation and adversity exposure. Development and Psychopathology, 32, 1788-1798.

Morin, E. L., Howell, B. R., Feczko, E., Earl, E., Pincus, M., Reding, K., ... Sanchez, M. M. (this issue). Developmental outcomes of early adverse care on amygdala functional connectivity in nonhuman primates. Development and Psychopathology, 32, 1579-1596.

Myrsten, A. L., Lundberg, U., Frankenhaeuser, M., Ryan, G., Dolphin, C., \& Cullen, J. (1984). Sex-role orientation as related to psychological and physiological responses during achievement and orthostatic stress. Motivation and Emotion, 8, 243-257.

Nachmias, M., Gunnar, M. R., Mangelsdorf, S., Parritz, R., \& Buss, K. A. (1996). Behavioral inhibition and stress reactivity: Moderating role of attachment security. Child Development, 67, 508-522.

Nelson, S. C., Syed, M., Tran, A. G. T. T., Hu, A. W., \& Lee, R. M. (2018). Pathways to ethnic racial identity development and psychological adjustment: The differential associations of cultural socialization by parents and peers. Developmental Psychology, 54, 2166-2180. doi:10.1037/dev0000597

Norona-Zhou, A. N., Morgan, A., Glynn, L. M., Sandman, C. A., Baram, T. Z., Stern, H. S., \& Davis, E. P. (2020). Unpredictable maternal behavior is associated with a blunted infant cortisol response. Developmental Psychobiology, $62,882-888$.

Obradović, J., \& Armstrong-Carter, E. (this issue). Addressing education inequalities and promoting learning through studies of stress physiology in elementary school students. Development and Psychopathology, 32, 1899-1913.

Parenteau, A. M., Alen, N. V., Deer, L. K., Nissen, A. T., Luck, A. T., \& Hostinar, C. E. (this issue). Parenting matters: Parents can reduce or amplify children's anxiety and cortisol responses to acute stress. Development and Psychopathology, 32, 1799-1809.

Perry, R. E., Braren, St. H., Opendak, H., Brandes-Aitken, M., Chopra, A., \& Woo, D., ... Family Life Project Key Investigators. (this issue). Elevated infant cortisol is necessary but not sufficient for transmission of environmental risk to infant social development: Cross-species evidence of mother-infant physiological social transmission. Development and Psychopathology, 32, 1696-1714.

Perry, N. B., DePasquale, C. E., Donzella, B., \& Gunnar, M. R. (this issue). Associations between stress reactivity and behavior problems for previously institutionalized youth across puberty. Development and Psychopathology, 32, 1854-1863.

Pollak, S. D., \& Wolfe, B. L. (this issue). How developmental neuroscience can help address the problem of child poverty. Development and Psychopathology, 32, 1640-1656.

Raby, K. L., Bernard, K., Gordon, K., \& Dozier, M. (this issue). Enhancing diurnal cortisol regulation among young children adopted internationally: A randomized controlled trial of a parenting-based intervention. Development and Psychopathology, 32, 1657-1668.

Reid, B., \& Danese, A. (this issue). Challenges in researching the immune pathways between early life adversity and psychopathology. Development and Psychopathology, 32, 1597-1624.

Russotti, J., Warmingham, J. M., Handley, E. D., Rogosch, F. A., \& Cicchetti, D. (this issue). Characterizing competence among a high-risk sample of emerging adults: Prospective predictions and biological considerations. Development and Psychopathology, 32, 1937-1953.

Rutter, M. (2016). Why is the topic of the biological embedding of experiences important for translation? Development and Psychobiology, 28, 1245-1258.

Sandman, C. A., Glynn, L. M., \& Davis, E. P. (2013). Is there a viabilityvulnerability tradeoff? Sex differences in fetal programming? Journal of Psychosomatic Research, 2013, 327-335.

Smotherman, W. P., \& Bell, R. W. (1980). Maternal mediation of early experience. In R. W. Bell \& W. P. Smotherman (Eds.), Maternal influences and early behavior (pp. 201-210). New York: Spectrum Puublications.

Steward, J. L., Towers, D. N., Coan, J. A., \& Allen, J. B. (2011). The oftneglected role of parietal EEG asymmetry and risk for major depressive disorder. Psychophysiology, 48, 82-95.

Stroud, L., Foster, E., Handwerger, K., Papandonatos, G. D., Granger, D., Kivlighan, K. T., \& Niaura, R. (2009). Stress response and the adolescent transition: Performance versus peer rejection stress. Development \& Psychopathology, 21, 47-68.

Stroud, L., Salovey, P., \& Epel, E. S. (2002). Sex differences in stress responses: Social rejection versus achievement stress. Biological Psychiatry, 52, 318-327.

Tang, A., Wade, M., Fox, N. A., Nelson, C. A., Zeanah, C. H., \& Slopen, N. (this issue). The prospective association between stressful life events and 
inflammation among adolescents with a history of early institutional rearing. Development and Psychopathology, 32, 1715-1724.

Tarullo, A. R., Tuladhar, C. T., Kao, K., Drury, E. B., \& Meyer, J. (this issue). Cortisol and socioeconomic status in early childhood: A multidimensional assessment. Development and Psychopathology, 32, 1876-1887.

Tottenham, N. (2020). Early adversity and the neotenous human brain. Biological Psychiatry, 87, 350-358.

Tottenham, N. (this issue). Neural meaning making, prediction, and prefrontal-subcortical development following early adverse caregiving. Development and Psychopathology, 32, 1563-1578.

Tottenham, N., Shapiro, M., Flannery, J., Caldera, C., \& Sullivan, R. M. (2019). Parental presence switches avoidance to attraction learning in children. Nature Human Behavior, 3, 1070-1077.

VanZomeren, A. A., Zhang, J., Lee, S., Gunlicks-Stoessel, M., Piehler, T., \& Cicchetti, D. (this issue). Timing, HPA axis functioning, multigenic risk, and depressive symptoms in African American youth: Differential associations without moderated mediation. Development and Psychopathology, 32, 1838-1853.

von Bertalanffy, L. (1969). General systems theory. New York: George Braziller. Wade, M., Sheridan, M. A., Zeanah, C. H., Fox, N. A., Nelson, C. A., \& McLaughlin, K. A. (this issue). Environmental determinants of physiological reactivity to stress: The interacting effects of early life deprivation, caregiving quality, and stressful life events. Development and Psychopathology, 32, 1732-1742.

Werner, E. E. (1996). Vulnerable but invincible: High risk children from birth to adulthood. European Child and Adolescent Psychiatry, 5, 47-51.

Werner, E. E., Bierman, J. M., \& French, F. E. (1971). The children of Kauai: A longitudinal study from the prenatal period to age ten. Honolulu: University of Hawaii Press.

Zijlmans, M. A., Riksen-Walraven, J. M., \& de Weerth, C. (2015). Associations between maternal prenatal cortisol concentrations and child outcomes: A systematic review. Neuroscience and Biobehavioral Review, 53, 1-24. 